\title{
Semiflexible Polymers in Shear Flow
}

\author{
Roland G. Winkler \\ Institut für Festkörperforschung, Forschungszentrum Jülich, D-52425 Jülich, Germany
}

(Received 20 June 2006; published 18 September 2006)

\begin{abstract}
The dynamics of semiflexible polymers under the influence of shear flow is studied analytically. Power laws are derived for various conformational and dynamical quantities which are in agreement with experimental findings. In particular, the tumbling motion is analyzed and expressions are provided for the probability distributions of the orientation angles and the tumbling time. The calculations explain the similarities in the behavior of flexible and semiflexible polymers as well as free-draining and nondraining systems.
\end{abstract}

DOI: $10.1103 /$ PhysRevLett.97.128301

PACS numbers: 83.80.Rs, 02.50.Ey, 05.40.Jc, 83.50.Ax

Experimental studies of individual DNA molecules in steady shear flow by fluorescence microscopy have provided a wealth of information on single polymer dynamics [1-6]. In particular, these experiments reveal remarkably large conformational changes due to tumbling motion; i.e., a polymer stretches and recoils in the coarse of time [1,5]. The dependence of the tumbling time on the shear rate (or Weissenberg number) was elucidated in Refs. [2,4]. The authors of Ref. [4] were even able to measure orientational distribution functions of $\lambda$-DNA. A number of theoretical studies have been performed in order to achieve a microscopic understanding of the observed phenomena [2,7-11], which provided various scaling relations for conformational and dynamical properties $[4,7,9,11]$.

Since shear flows are omnipresent in biological systems and technical applications, e.g., microfluidics, the understanding of the dynamics of semiflexible polymers - such as DNA-is of great practical interest. The microscopic conformational properties affect the macroscopic rheological behavior of the polymer solution, and hence a detailed theoretical description of the microscopic dynamics is desirable.

The dynamic behavior of a macromolecule in shear flow is governed by various parameters; aside from the shear rate, the finite chain extensibility is of major importance. To what extent hydrodynamic interactions, thermal fluctuations, and chain persistence play a role has not yet been explored, although some results for chain extension have been presented [12-14].

In this Letter, the dynamics of a semiflexible polymer in shear flow is studied taking into account hydrodynamic interactions. An analytical expression is derived for the orientational distribution function, and the dependence of the tumbling time on shear rate is determined. It will be shown that these quantities agree almost quantitatively with experimental results. As it turns out, hydrodynamic interactions play a minor role only, as long as the Weissenberg number is used in data presentation.

The steady state dynamics will be analyzed only, where thermal fluctuations determine the system behavior. Hence, relevant dynamical information will be extracted from ensemble averages. This demonstrates that valuable insight into the system behavior can be obtained from ensemble averages and not only from the dynamics of individual molecules [10].

The analytical results show that due to shear flow high order correlations in time and the whole history of time evolution of the system are important for structural as well as dynamical quantities. This is the origin of what is called intermittency phenomena in Ref. [4] - characterized by algebraic or exponential tails of distribution functionsalthough the underlying thermal process is Gaussian and Markovian and the equations of motion are linear. Thus, a polymer in shear flow is an example where a very complex system behavior is obtained despite the underlaying simple Gaussian process [15].

The dynamics of macromolecules in shear flow is analyzed using a Gaussian semiflexible polymer model [16]. This model proved adequate in studies of equilibrium $[17,18]$ and nonequilibrium [19] dynamical aspects of DNA in solution. The polymer of length $L$ is described by a continuous, differentiable space curve $\boldsymbol{r}(s, t)$, where $s$ is the contour coordinate along the chain $(-L / 2<s<$ $L / 2$ ) [18]. The equation of motion, including hydrodynamic interactions, is given by the Langevin equation [17-19]

$$
\begin{aligned}
\frac{\partial}{\partial t} \boldsymbol{r}(s, t)= & \int_{-L / 2}^{L / 2} \mathbf{H}\left(s, s^{\prime}\right)\left[2 \nu k_{B} T \frac{\partial^{2}}{\partial s^{\prime 2}} \boldsymbol{r}\left(s^{\prime}, t\right)\right. \\
& \left.-\epsilon k_{B} T \frac{\partial^{4}}{\partial s^{\prime 4}} \boldsymbol{r}\left(s^{\prime}, t\right)+\boldsymbol{\Gamma}\left(s^{\prime}, t\right)\right] d s^{\prime}+\mathbf{K} \boldsymbol{r}(s, t),
\end{aligned}
$$

with appropriate boundary conditions [17]. The term with the second derivative in $\boldsymbol{r}$ captures the chain flexibility; i.e., it takes chain entropy into account. The term with the fourth derivative accounts for bending stiffness. Note that no excluded volume interactions are taken into account, which may affect the system behavior for small and intermediate Weissenberg numbers. The stochastic force $\boldsymbol{\Gamma}(s, t)$ is assumed to be stationary, Markovian, and Gaussian with zero mean. The shear rate tensor $\mathbf{K}$ possesses only one 
nonzero element: $K_{x y}=\dot{\gamma}$, where $\dot{\gamma}$ is the shear rate. $k_{B}$ is the Boltzmann factor, $T$ the temperature, $\epsilon=3 /(4 p)$, where $p$ is related to the chain persistence length $\left(l_{p}\right)$ via $p=1 /\left(2 l_{p}\right)$, and $\nu=3 p \mu / 2$ [16-19]. The factor $\mu$ is determined from the constraint for the contour length $\int_{-L / 2}^{L / 2}\left\langle[\partial \boldsymbol{r} / \partial s]^{2}\right\rangle d s=L$ [16-19], where $\mu=1$ at equilibrium. Hydrodynamic interactions are taken into account by the Rotne-Prager tensor and treated within the preaveraging approximation [18]. For averaging, the equilibrium distribution function without shear is used. This is certainly an approximation which does not apply for all shear rates equally well, but it will provide some insight into the influence of hydrodynamic interactions on chain dynamics. The approach neglects the effect of polymer deformation on hydrodynamic interactions; hence, subtle effects on conformations and dynamical quantities due to timedependent variations of the hydrodynamic interactions cannot be captured. Equation (1) is then a linear equation with coefficients depending in a nonlinear manner on the shear rate. By the eigenfunction expansion $\boldsymbol{r}(s, t)=$ $\sum_{n=0}^{\infty} \boldsymbol{X}_{n}(t) \psi_{n}(s)$ (for the eigenfunction cf. Refs. [17,18]), Eq. (1) turns into

$$
\frac{d}{d t} \boldsymbol{\chi}_{n}(t)=-\frac{1}{\tilde{\tau}_{n}} \boldsymbol{\chi}_{n}(t)+\sqrt{\frac{k_{B} T \tau_{n}}{3 \pi \eta \tilde{\tau}_{n}}} \boldsymbol{\Gamma}_{n}(t)+\mathbf{K} \boldsymbol{\chi}_{n}(t),
$$

where $\eta$ is the viscosity of the solvent, $\tilde{\tau}_{n}$ the $n$th relaxation time with hydrodynamic interactions, $\tau_{n}$ the $n$th freedraining relaxation time, and $\boldsymbol{\Gamma}_{n}(t)$ the $n$th stochastic force amplitude $[17,18]$. The relaxation times $\tau_{n}$ are related to the wave numbers $\zeta_{n}$ of the eigenvalue equation by

$$
\tau_{n}=\frac{\pi \eta}{k_{B} T p \mu\left[\zeta_{n}^{2}+\zeta_{n}^{4} /\left(4 p^{2} \mu\right)\right]},
$$

where $\zeta_{n}=n \pi / L$ in the limit of a flexible polymer [18], i.e., $p L \rightarrow \infty$. The shear rate tensor $\mathbf{K}$ couples $d \chi_{n}^{x}(t) / d t$ to $\chi_{n}^{y}(t)$, and hence the whole time history of the latter is important for the time evolution of $\chi_{n}^{x}(t)$.

Using the eigenfunction expansion, a general expression is obtained easily for the parameter $\mu$ containing the sum over all modes. With Eq. (3), in the limit $p L \gg 1$, and the ratio $\tilde{\tau}_{n} / \tilde{\tau}_{1}^{0}=\left(1+\pi^{2} /(2 p L)^{2}\right) /\left(\mu n^{2}\right)$ following for $\mu \gg$ 1 , where $\tilde{\tau}_{1}^{0}$ is the longest relaxation time $\tilde{\tau}_{1}$ at $\mu=1$, this expression reduces to

$$
\mu^{3}-\mu^{5 / 2}-\frac{\left\{1+[\pi /(2 p L)]^{2}\right\}^{2} \pi^{4}}{540 p L} \mathrm{Wi}^{2}=0 .
$$

Here, the Weissenberg number $\mathrm{Wi}=\dot{\gamma} \tilde{\tau}_{1}^{0}$ is introduced. The solution of this equation deviates from the full expression by a few percent only for all Wi as long as $p L>$ 2 ; i.e., the approximate expression applies even to rather stiff polymers. The reason is that in the derivation of Eq. (4) bending modes do not play a major role. Only the lowest modes contribute to the overall system behavior. For Wi $\gg 1$, Eq. (4) yields

$$
\mu \approx \mathrm{Wi}^{2 / 3} \sqrt[3]{\pi^{4} /(540 p L)} .
$$

This relation is an important result of this Letter, because the factor $\mu$ appears in the relaxation times, and hence in all other quantities, and determines the system behavior at large Weissenberg numbers. It is important to note that $\mu$ is only weakly dependent on the persistence length and does not depend on hydrodynamic interactions. Equation (4) suggests that for semiflexible polymers $(p L>2)$ a length and persistence length independent behavior is obtained if (measured) quantities are presented in terms of $\mathrm{Wi} / \sqrt{p L}$. I like to point out that even for rodlike polymers the dependence $\mu \sim \mathrm{Wi}^{2 / 3}(\mathrm{Wi} \gg 1)$ is observed.

Equation (3) clearly shows that an increasing $\mu$ leads to decreasing relaxation times; i.e., all processes become faster. In addition, the contribution of bending modes decreases. In general, a polymer of a given length behaves increasingly flexible with increasing shear rate.

The conformational properties can conveniently be characterized by the average gyration tensor [9]. Its component in the flow direction $\left(G_{x x}\right)$ increases with increasing shear rate from the equilibrium value and reaches a plateau in the limit $\mathrm{Wi} \rightarrow \infty$, which is given by $G_{x x}^{m} \approx 90 L^{2} / 945$ using the approximations leading to Eq. (4). The way in which the maximum value is asymptotically reached is governed by $\mu$. With $\mu$ from Eq. (5), the calculation yields the dependence $\left(G_{x x}^{m}-G_{x x}\right) / G_{x x}^{m}=1 / \sqrt{\mu} \sim \mathrm{Wi}^{-1 / 3}$. The same dependence for the approach to full extension of a wormlike tethered chain has been found in Ref. [20] by scaling arguments, simulations, and from measurements.

The calculation of the gyration tensor transverse to the flow direction yields $G_{y y}=G_{z z}=k_{B} T /(\pi \eta L) \sum_{n=1}^{\infty} \tau_{n}$, which turns into $G_{y y} \sim \mu^{-1}$ for large Wi, and hence the thickness $\sqrt{G_{y y}}$ transverse to the flow direction decreases as $\sqrt{G_{y y}} \sim \mathrm{Wi}^{-1 / 3}$ in the limit $\mathrm{Wi} \rightarrow \infty$. Scaling arguments predict the dependencies $\mathrm{Wi}^{-1 / 4}$ and $\mathrm{Wi}^{-3 / 11}$ $[9,21]$, i.e., exponents with a magnitude somewhat smaller than that of the current model. The experimental results presented in Ref. [9] do not provide a unambiguous exponent and can be considered consistent with the current theoretical dependence.

A polymer is not only deformed but also aligned in shear flow. The authors of Ref. [4] provided a very valuable contribution to the understanding of the alignment by measuring the probability distribution functions of the orientation angles. Such a probability distribution function can also be calculated within the current approach. The distribution function of the end-to-end vector $\boldsymbol{r}_{e}=$ $\left(x_{e}, y_{e}, z_{e}\right)^{T}$ is given by the Gaussian [11]

$$
P\left(\boldsymbol{r}_{e}\right)=\exp \left(-\frac{1}{2} \boldsymbol{r}_{e}^{T} \mathbf{A}^{-1} \boldsymbol{r}_{e}^{T}\right) /\left[(2 \pi)^{3 / 2} \sqrt{|\mathbf{A}|}\right],
$$

where the matrix A can easily be obtained from the correlation functions $\left\langle\chi_{n}^{\alpha} \chi_{n}^{\beta}\right\rangle$. Integration over the magnitude of $\boldsymbol{r}_{e}$ yields the distribution function $P(\vartheta, \varphi)$, where $\vartheta$ is the 
angle between $\boldsymbol{r}_{e}$ and its projection in the $x y$ plane and $\varphi$ is the angle between the projection and the $x$ axis (cf. Ref. [4]). Further (analytical) integration with respect to $\vartheta$ yields the distribution function $P(\varphi)$. Using the approximations indicated before Eq. (4), the latter reads

$$
P(\varphi)=\frac{\sqrt{\pi^{4}+4 \widetilde{\mathrm{Wi}}^{2}\left(\pi^{2}-4\right) / \mu^{2}}}{2 \pi\left[\pi^{2}+4 \widetilde{\mathrm{Wi}}^{2} \sin ^{2}(\varphi) / \mu^{2}-4 \widetilde{\mathrm{Wi}} \sin (2 \varphi) / \mu\right]},
$$

with the abbreviation $\widetilde{\mathrm{Wi}}=\mathrm{Wi}\left\{1+[\pi /(2 p L)]^{2}\right\}$. Equation (7) accounts for the full distribution function within a relative error of $4 \%$ only for $p L \geq 5$ and $\mathrm{Wi}>1$.

The distribution functions $P(\vartheta, \varphi)$ and $P(\varphi)$ exhibit features already obtained for a dumbbell model of a polymer [11]; however, the above expressions are more general and include the dependence on polymer length and persistence length. Naturally, the expressions exhibit the asymptotic power-law dependencies $P(\vartheta) \sim|\vartheta|^{-2}$ and $P(\varphi) \sim(\sin \varphi)^{-2}$ in the limit of sufficiently large angles $[4,11]$. In contrast to the considerations in Ref. [22], where the dependence $P(\vartheta) \sim|\vartheta|^{-2}$ is attributed to the deterministic rotational motion of the polymer, the current distribution functions are calculated in the steady state, where the system behavior is governed by thermal fluctuations. Hence, the universal aspects of the orientational distribution functions are independent of the particular source of random excitations - they rather depend on the statistical properties of the noise only [4].

The maximum of the distribution function $P(\varphi)$ follows from the equation $\tan \left(2 \varphi_{m}\right)=2 \mu / \mathrm{Wi}$, where $\varphi_{m}$ denotes the angle at the maximum. The calculation of the orientational angle $\left(\chi_{G}\right)$ in terms of the components of the gyration tensor $\left[\tan \left(2 \chi_{G}\right)=2 G_{x y} /\left(G_{x x}-G_{y y}\right)\right]$ [2] yields the same dependence. With Eq. (5), the angle at the maximum is given by $\varphi_{m}=\sqrt[3]{\pi^{4} /(540 p L)} \mathrm{Wi}^{-1 / 3}$ for $\mathrm{Wi} \gg 1$. The dependence on the Weissenberg number is in agreement with computer simulation results [2]. Taking typical values for $\lambda$-DNA, the magnitude of the slope is larger for intermediate $\mathrm{Wi}$, consistent with experimental findings [2].

The calculation of the width $(\Delta \varphi)$ of the distribution function (7) at half height yields $\Delta \varphi \sim \mathrm{Wi}^{-1}$ in the limit $\mathrm{Wi} \rightarrow 0$. Since in this regime $\mu=1$, the distribution function $P(\varphi)$ is essentially independent of the persistence length $(p L \gg \pi / 2)$; i.e., a universal distribution function is obtained independent of any specificity of the polymer. For Wi $\gg 1$ follows the relation $\Delta \varphi=\sqrt{\pi^{2}-4} \mu / \widetilde{\mathrm{Wi}}$, which yields $\Delta \varphi \sim \mathrm{Wi}^{-1 / 3}(p L)^{-1 / 3}$ for sufficiently large $p L$. The same dependence with respect to the Weissenberg number has been obtained in Refs. [4,11,22]. In addition, the model yields a power-law dependence on the polymer length and persistence length. This result is completely different from the term provided in [4], where $\Delta \varphi$ is independent of $L$ and $l_{p}$.
Figure 1 displays the probability density function $P(\varphi)$ for $\lambda$-DNA. The only parameters appearing in the theoretical description are the product $p L$, the thickness of the DNA, and the Weissenberg number. Using the parameters of Ref. [4], namely, $L=21 \mu \mathrm{m}$ and the radius of gyration $r_{g}^{2}=0.53 \mu \mathrm{m}^{2}$, the DNA persistence length is $l_{p} \approx$ $70 \mathrm{~nm}$, i.e., $p L=L /\left(2 l_{p}\right)=150$. The DNA thickness is set to $d=2 \mathrm{~nm}$. Applying the experimental Weissenberg number $\mathrm{Wi}=25$ leads to a pronounced mismatch between the measurements (symbols) and the theoretical distribution function. Only by using a 4 times larger Wi, i.e., $\mathrm{Wi}=$ 100 in the theoretical model, the very good agreement of Fig. 1 is obtained. I attribute this necessary adjustment to differences in the longest measured and theoretical relaxation times, respectively, used in the definition of Wi. The inset of Fig. 1 shows the width of $P(\varphi)$ at half height for various Weissenberg numbers. The theoretical curve reproduces the experimentally obtained dependence very well, when again 4 times larger theoretical Wi values are used.

Insight into the dynamical behavior of semiflexible polymers can be gained by the tumbling time [1,4]. Since Eq. (1) is a Gaussian but non-Markovian process in general, it is difficult to extract an analytical expression for such a time. Tumbling is related to crossings of planes parallel to the $x z$ plane, or, expressed in terms of the angle $\varphi$, the plane $\varphi=0$, by an orientational vector, e.g., the end-to-end vector. Hence, a characteristic time can be defined by considering successive conformations where $y_{e}$ is zero. This strategy is similar to a definition where the angular dynamics is considered [7]. To study the dynamics of $y_{e}$ has the advantage that the normal mode

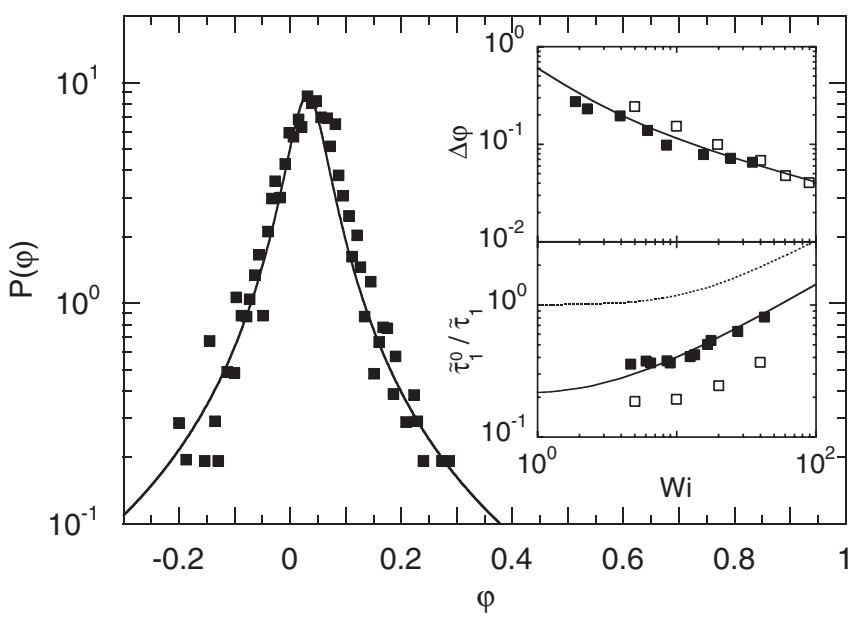

FIG. 1. Comparison of the theoretical probability density function of the angle $\varphi$ (solid line) with experimental results on $\lambda$-DNA for the Weissenberg number $\mathrm{Wi}=25$ [4]. The top inset displays the width at half height of $P(\varphi)$ and the bottom inset shows the tumbling time $t_{T}=\tilde{\tau}_{1}$ for the theoretical model (solid line), experiments ( $\square$ ), and simulations ( $\square$ ) [4,7]. The dotted line is $\mu(\mathrm{Wi})$. For the comparison, the theoretical Weissenberg numbers are set to 4 times the displayed experimental values. 
amplitudes $\chi_{n}^{y}$ constitute a Gaussian and Markovian process with the correlation functions $\left\langle\chi_{n}^{y}(t) \chi_{n}^{y}(0)\right\rangle=$ $k_{B} T \tau_{n} /(3 \pi \eta) \exp \left(-t / \tilde{\tau}_{n}\right)$ in the stationary state. For $p L>5$, the correlation function for $y_{e}$ is well approximated by the mode $n=1$ only, i.e., $\left\langle y_{e}(t) y_{e}(0)\right\rangle \approx$ $\left\langle y_{e}(0)^{2}\right\rangle \exp \left(-t / \tilde{\tau}_{1}\right)$, and thus is approximately a Gaussian and Markovian process. The probability $P(t)$ for not crossing the plane defined by $\varphi=0$ is then decaying exponentially for large times [15] with the characteristic time $t_{T}=\tilde{\tau}_{1}$, which is identified as the tumbling time $t_{T}$. Hence, $\tilde{\tau}_{1}^{0} / \tilde{\tau}_{1}=\tilde{\tau}_{1}^{0} / t_{T}=\mu$, which means that the tumbling time is determined by the force along the polymer contour exerted by the flow. Consequently, the force can be directly measured via the tumbling time.

The authors of Ref. [4] have been able to measure the probability density function of the elongation tumbling time and determined characteristic tumbling times. The bottom inset of Fig. 1 compares the measured values and simulation results [4] with the theoretical prediction. Taking 4 times large theoretical Weissenberg numbers and dividing $\tilde{\tau}_{1}^{0} / \tilde{\tau}_{1}$ by five instead of four, the latter adjustment might be related to differences in the definition of the tumbling time, the theoretical curve agrees qualitatively with the measured data. The comparison shows that the experimental values are in a crossover regime with respect to the Weissenberg number. The limiting behavior $t_{T} \sim$ $\mu^{-1} \sim \mathrm{Wi}^{-2 / 3}$, follows for larger Wi. The power-law dependence is consistent with the scaling predictions of Refs. [4,22]. In addition, however, the current approach predicts also a dependence on the chain and persistence length [cf. Eq. (5)].

The present model does not predict any periodic (tumbling) motion [4] in contrast to measurements and simulations of the power spectral densities (PSDs) of polymer orientation [2] or correlations [10], respectively. Neither the PSD of the end-to-end distance or the radius of gyration in flow direction nor cross correlation functions among their spacial components exhibit a maximum and thus no deterministic cycle in the stationary state seems to be present. (The PSDs of the polymer orientation [2] or correlations among extensions [10] cannot be calculated analytically due to appearing nonlinearities.) However, the measured peak frequencies exhibit the same power-law dependence on the Weissenberg number as the theoretical tumbling time. Moreover, the simulations of Ref. [10] show that the longest relaxation time of a polymer exhibits the same dependence on the Weissenberg number as the extracted tumbling time, which is in agreement with the present theoretical analysis.

In summary, the semiflexible polymer model provides a consistent description of the structural and dynamical properties of DNA in shear flow. In particular, it shows essentially no dependence on hydrodynamic interactions. The influence of the latter on polymer dynamics is ad- sorbed in the Weissenberg number. Thus, only a very weak dependence on hydrodynamic interactions is expected for models which take it into account in more detail. A weak dependence on the persistence length is predicted for various quantities. This weak dependence complicates the measurement of DNA parameters by shear experiments. I hope that the presented results will stimulate further experimental studies on DNA molecules to gain deeper insight into length and persistence length specific features of distribution functions and characteristic times.

Financial support by the Deutsche Forschungsgemeinschaft within the Priority Program SPP 1164 is gratefully acknowledged.

[1] D. E. Smith, H. P. Babcock, and S. Chu, Science 283, 1724 (1999).

[2] C. M. Schroeder, R.E. Teixeira, E. S. G. Shaqfeh, and S. Chu, Phys. Rev. Lett. 95, 018301 (2005).

[3] R.E. Teixeira, H.P. Babcock, E.S. G. Shaqfeh, and S. Chu, Macromolecules 38, 581 (2005).

[4] S. Gerashchenko and V. Steinberg, Phys. Rev. Lett. 96, 038304 (2006).

[5] P. LeDuc, C. Haber, G. Boa, and D. Wirtz, Nature (London) 399, 564 (1999).

[6] P. S. Doyle, B. Ladoux, and J.-L. Viovy, Phys. Rev. Lett. 84, 4769 (2000).

[7] A. Celani, A. Puliafito, and K. Turitsyn, Europhys. Lett. 70, 464 (2005).

[8] N. J. Woo, E. S. G. Shaqfeh, and B. Khomami, J. Rheol. (N.Y.) 48, 299 (2004).

[9] C. M. Schroeder, R.E. Teixeira, E. S. G. Shaqfeh, and S. Chu, Macromolecules 38, 1967 (2005).

[10] R. Delgado-Buscalioni, Phys. Rev. Lett. 96, 088303 (2006).

[11] A. Puliafito and K. Turitsyn, Physica (Amsterdam) 211D, 9 (2005).

[12] A. Dua and B. J. Cherayil, J. Chem. Phys. 113, 10776 (2000).

[13] X. Wang and A.P. Chatterjee, Macromolecules 34, 1118 (2001).

[14] R.M. Jendrejack, J.J. de Pablo, and M.D. Graham, J. Chem. Phys. 116, 7752 (2002).

[15] S. N. Majumdar and C. Sire, Phys. Rev. Lett. 77, 1420 (1996).

[16] R. G. Winkler, J. Chem. Phys. 118, 2919 (2003).

[17] R. G. Winkler, S. Keller, and J. O. Rädler, Phys. Rev. E 73, 041919 (2006).

[18] L. Harnau, R. G. Winkler, and P. Reineker, J. Chem. Phys. 104, 6355 (1996).

[19] R. G. Winkler, Phys. Rev. Lett. 82, 1843 (1999).

[20] B. Ladoux and P. S. Doyle, Europhys. Lett. 52, 511 (2000).

[21] P.S. Doyle, E.S. G. Shaqfeh, and A.P. Gast, J. Fluid Mech. 334, 251 (1997).

[22] M. Chertkov, I. Kolokolov, V. Lebedev, and K. Turitsyn, J. Fluid Mech. 531, 251 (2005). 